\title{
Pandemic influenza A (H1N1) 2009: the experience of the first six months
}

\author{
Jean Maritz*, Leana Maree* and Wolfgang Preiser \\ Division of Medical Virology, Department of Pathology, \\ National Health Laboratory Service and University of \\ Stellenbosch, Cape Town, South Africa
}

\begin{abstract}
After a break of 41 years, 2009 saw the first influenza pandemic of the 21 st century caused by a triple-reassortant influenza A (H1N1) virus. The current estimated case fatality rate is lower than that of previous influenza pandemics, but this may change as the pandemic evolves. Illness frequently occurs in previously healthy, young adults with a wide range of clinical presentations. The majority of circulating pandemic viruses remain susceptible to neuraminidase inhibitors, although all strains are intrinsically resistant to the adamantanes. Monovalent vaccines against the pandemic strain are available in both live attenuated and inactivated forms. This review aims to summarise important virological, epidemiological and clinical aspects of the pandemic influenza A (H1N1) virus for physicians and other clinical personnel. Clin Chem Lab Med 2010;48:11-21.
\end{abstract}

Keywords: H1N1; influenza A virus; pandemic; review; swine flu.

\section{Introduction}

On 11 June 2009, only 2 months after the first human infections with a novel influenza A (H1N1) virus of swine origin were reported from Mexico and the USA (1), the World Health Organization (WHO) declared the first influenza pandemic of the 21 st century (2).

Within weeks, the pandemic influenza A (H1N1) 2009 virus [from here on referred to as pandemic H1N1/09 virus (3)], a triple-reassortant swine influenza virus, had spread across much of the globe. As of 15 November 2009, more than 526,060 cases of pandemic H1N1/09 infection have

*Corresponding authors: Jean Maritz, MBChB MSc and Leana Maree, MBChB, Division of Medical Virology, Department of Pathology, National Health Laboratory Service and University of Stellenbosch, Cape Town 7505, South Africa

Phone: +27 21938 9347, Fax: +27 21938 9361,

E-mail: jean.maritz@gmail.com; leana@sun.ac.za

Received November 21, 2009; accepted November 23, 2009;

previously published online December 22, 2009 been reported worldwide from over 206 countries and territories, with at least 6770 deaths (4). Since it is no longer required to confirm or report all clinically diagnosed cases, this figure significantly underestimates the true number of cases.

Influenza A viruses are well recognised for their potential to cause pandemics, which has happened three times during the 20th century (5). The highly pathogenic avian influenza (HPAI) A (H5N1) virus re-emerged at the end of 2003, and has since spread over wide areas of Asia, Europe, and Africa, causing sporadic but severe human cases (442 cases with 262 deaths as of 24 September 2009) (6). As a consequence, pandemic preparedness has been strengthened significantly over the past years. The 2009 pandemic is putting these measures to the test - although the new virus is thus far not highly virulent, the outbreak has caused considerable disruption worldwide. Clinical findings vary from asymptomatic infection to acute illness progressing to acute respiratory distress syndrome, frequently in young patients (7-9). As the influenza season has now come to an end in the southern hemisphere, the upcoming influenza season in the northern hemisphere will pose a challenge to healthcare services.

This review is based on the knowledge gained during the first 6 months of the pandemic, and aims to summarise important virological, epidemiological and clinical aspects of the pandemic H1N1/09 virus. It reflects the current state of knowledge, and as the pandemic evolves and more scientific studies are conducted, some of the statements made might soon be obsolete.

\section{Virology}

Influenza A viruses are negative-sense, single-stranded RNA viruses that belong to the family Orthomyxoviridae. Their genome is divided into eight segments, so that concurrent infection with two different strains can lead to reassortment, producing new viral strains. The envelope glycoproteins, haemagglutinin (HA) and neuraminidase (NA), are the major antigenic determinants of the cellular and humoral immune response to infection. They are also used to subtype influenza A viruses into $16 \mathrm{HA}$ and nine NA subtypes. Aquatic birds are the natural reservoir of all influenza A virus subtypes, but only $\mathrm{H} 1, \mathrm{H} 2$ and $\mathrm{H} 3$, and $\mathrm{N} 1$ and $\mathrm{N} 2$ subtypes have become established in human beings.

Phylogenetic analyses of the pandemic H1N1/2009 virus show that six genome segments stem from a triple-reassor- 
tant virus circulating in North American swine, seeded from human H3N2 (PB1), avian (PB2 and PA) and classical swine (HA, NP and NS) lineages. The neuraminidase and matrix gene segments are most closely related to the Eurasian avianlike swine H1N1 lineage (Figure 1) $(10,11)$. The high sequence identity $(99.9 \%)$ within each gene segment between isolates of the pandemic $\mathrm{H} 1 \mathrm{~N} 1 / 09$ virus suggest that the inter-species transmission was a single event or multiple events involving closely related viruses relatively recently (11).

The new virus may have been circulating undetected in swine herds for several years, as the closest ancestors for all eight segments are of swine origin and surveillance for swine influenza viruses is poor. However, although pandemic H1N1/09 infection has been described in swine herds and occasionally in other animal species in various countries, evidence points towards transmission from humans to pigs, i.e., reverse zoonoses (12).

\section{Pathogenesis}

Several genes are involved in determining influenza virus host- and tissue specificity, as well as pathogenicity, including HA, PB2, PB1-F2 and NS1 $(13,14)$. Sequence analyses of pandemic H1N1/09 virus isolates do not show genetic markers associated with high pathogenicity or increased transmissibility (11). This suggests that unknown genetic determinants may be responsible for the efficient transmission and replication of pandemic H1N1/09 virus in humans.

Pandemic H1N1/09 virus pathogenicity and transmissibility studies conducted in ferrets, mice and cynomolgus macaques proved that the pandemic strain is more pathogenic than seasonal H1N1 influenza, as the virus replicated more efficiently in the lungs of all three animal models (15-17). Macaque lungs demonstrated severe thickening of the alve- olar walls, and the alveolar spaces were filled with inflammatory infiltrates and oedematous exudates. Both type I and type II pneumocytes were infected. Ferrets inoculated with pandemic H1N1/09 virus displayed more severe symptoms, involving the entire respiratory tract, compared with ferrets inoculated with seasonal influenza A (H1N1) which displayed symptoms of necrotising rhinitis only (17). The detection of pandemic H1N1/09 virus in the intestinal tract of some infected ferrets is consistent with the gastrointestinal complaints from several human patients with pandemic H1N1/09 infection (16).

There is conflicting evidence regarding the transmissibility of pandemic H1N1/09 in animal models. Two of the studies found that it spreads as efficiently as seasonal influenza A (H1N1) via aerosols and respiratory droplets $(15,17)$. However, one study found that pandemic H1N1/09 virus is transmitted less efficiently via respiratory droplets (16). Droplet transmission does appear to be effective in humans, as an outbreak of pandemic H1N1/09 among tourists in China was caused by talking with the index patient at close range (18).

There is very limited data available regarding the systemic pathology of pandemic H1N1/09 virus infection in humans. Only one case series on the autopsy findings of 21 confirmed infections in Brazilian patients has been published to date (19). Macroscopically, the lungs were diffusely oedematous with varying degrees of haemorrhage. Microscopically, three distinct patterns of pulmonary changes were identified: 1) exudative diffuse alveolar damage, with alveolar fibrinous exudates and oedema, interstitial oedema and reactive pneumocytes; 2) exudative diffuse alveolar damage with severe haemorrhage; and 3) severe necrotising bronchiolitis with extensive bronchiolar wall necrosis and dense neutrophilic infiltrates. None of the other organs that were examined showed signs of direct viral injury, and any changes in these organs were due to secondary organ failure.

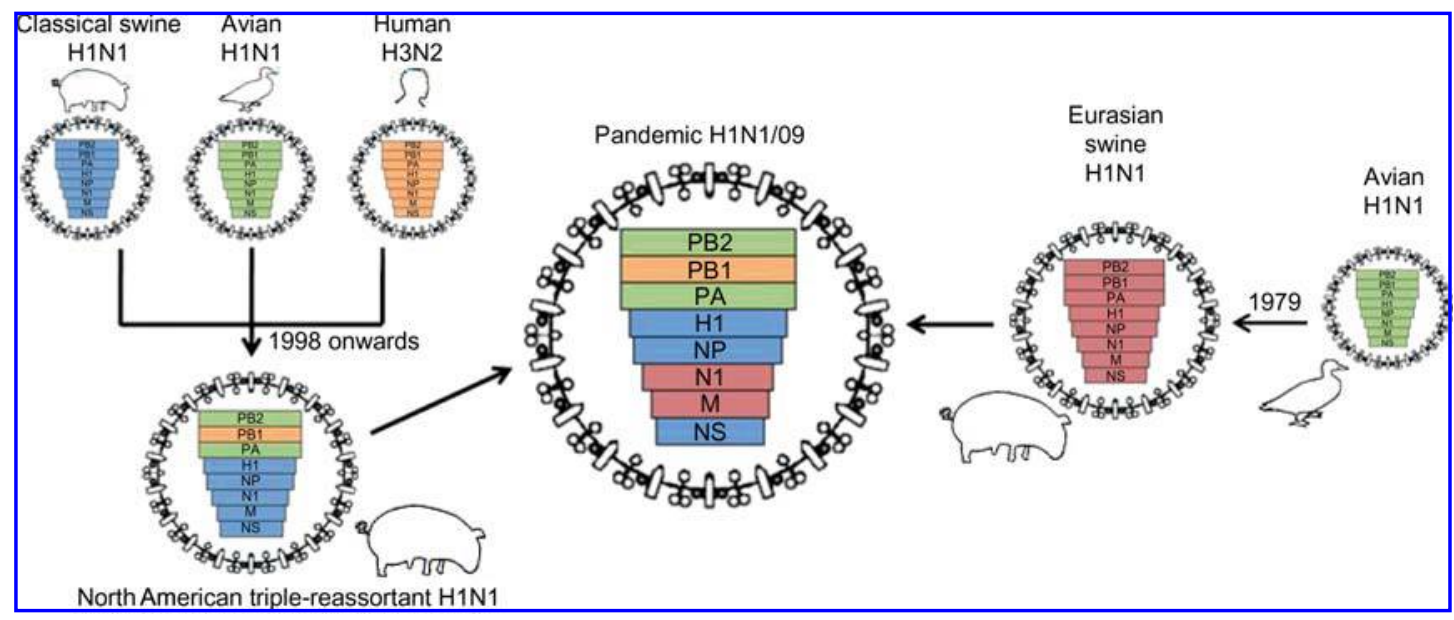

Figure 1 Origin of the pandemic H1N1/09 virus.

It contains gene segments most closely related to those from triple-reassortant North American H1N1 swine influenza virus and Eurasian swine influenza virus. It is unknown when the latest reassortment event took place. 


\section{Epidemiology}

\section{Basic reproductive number $\left(\mathbf{R}_{0}\right)$}

$\mathrm{R}_{0}$ is an estimate of the average number of secondary cases generated per primary case and needs to exceed 1 to sustain an epidemic. The $\mathrm{R}_{0}$ for pandemic $\mathrm{H} 1 \mathrm{~N} 1 / 09$ has been reported to range from 1.37 to 2.3 (20-22). In Japan, a minorspecific $R_{0}$ was calculated to be 2.8 , suggesting that a population of minors can sustain transmission of the pandemic H1N1/09 strain amongst themselves (22). These reported estimates are greater than the mean reproductive number for seasonal influenza epidemics spanning three decades in temperate European countries, estimated at 1.3 (23).

Factors unrelated to the causative agent of an epidemic may also influence the perceived $\mathrm{R}_{0}$. These include the use of antiviral medications, the specific point during the epidemic at which the $\mathrm{R}_{0}$ is reported, public awareness with utilisation of health services or reporting systems, as well as the presence of large clusters. The $\mathrm{R}_{0}$ may be overestimated if large clusters, such as schools, day care centres or old age homes are included, and should therefore be interpreted with caution (20).

\section{Secondary attack rate}

The secondary attack rate in households has been estimated to range from $7.6 \%$ to $33 \%$ (24-26), and was significantly higher among siblings than other family members (24). Social distancing and school closures can theoretically reduce peak influenza attack rates by $40 \%-50 \%$, especially when used in combination with prophylactic treatment (27, 28).

\section{Pre-existing immunity and age distribution}

Older individuals may have a degree of cross protection conferred by neutralising antibodies directed against other influenza A viruses, as suggested by serologic studies demonstrating higher antibody titres in individuals born before 1950 (29). This is supported by the higher frequency of disease in younger individuals, even in the absence of obvious large clusters (20). Despite this, the median age of cases positive for pandemic H1N1/09 in Australia was not much different from seasonal H1N1 infection during 2007 and 2008 (21 vs. 23 years). Seasonal influenza A (H3N2) infection, which dominated in previous years, tends to occur in older people (median of 33 years in 2008 in the same population). Therefore, the dominating seasonal strain needs to be taken into account when comparing the age distribution of cases of seasonal and pandemic strains (30).

Approximately $7 \%$ of adults and one-third of those above 65 years of age have protective levels of pre-existing antibodies, with the highest titres of antibodies found in subjects born before 1930, who were likely exposed to the 1918-like H1N1 virus $(29,31)$. It is also possible that heterotypic immunity from antibodies against neuraminidase or cellular immune responses may contribute to the protection in older adults (29). The younger median age of cases of the current pandemic may also reflect differences in social networking between age groups, and case-ascertainment bias may also account for differences in observed rates, as young patients may present more readily for testing (32).

\section{Spread}

During the influenza season in the southern hemisphere, selected countries reported laboratory based incidence rates ranging from 25 to 75/100,000 population (33-35). Sentinel surveillance in Australia reported that more than $90 \%$ of influenza-like illness cases were positive for the pandemic strain by the 8 th week of surveillance, with a peak of $95 \%$ by the 10th week (30). This was comparable to surveillance results from New Zealand and South Africa that showed an epidemic peak 4-8 weeks after the first reported case, and continuing for about 4 weeks $(34,35)$.

\section{Clinical characteristics}

\section{Age}

Pandemic H1N1/09 has been shown to affect all age groups, with a preponderance of younger cases. Notification rates in New Zealand were notably higher in those under 1 year of age and those aged 15-29 years (219/100,000 and 125/ 100,000 , respectively) compared with older individuals (35), consistent with the demonstrated lack of pre-existing antibodies in children and young adults (29).

The median age from various reporting countries ranges from 13 years to 23 years $(20,30,33,34,36)$. In South Africa, $64 \%$ of cases were under the age of 20 years (34), and in North America, $60 \%$ of all identified cases were under the age of 18 years (32). Some countries reported only $5 \%-8 \%$ of cases to be older than 50 years $(32,36)$.

Reports on hospitalised patients (with confirmed or probable infection) indicated a median age ranging from 31 to 44 years (36), with up to $90 \%$ of admitted patients under 52 $(7,8)$ and up to $18 \%$ under 5 years of age $(32)$.

The median age of patients admitted to an intensive care unit (ICU) was 32-43 years $(36,37)$. Comparing the median age of hospitalised and non-hospitalised cases revealed that the median age of patients requiring admission is higher than that of non-hospitalised cases. However, infants $(<1$ year of age) had the highest age specific incidence of ICU admission in New Zealand (38).

\section{Signs and symptoms}

Presenting symptoms were most commonly fever (91\%$100 \%)$, respiratory symptoms $(92 \%-100 \%)$, sore throat $(66 \%-77 \%)$ generalised weakness $(56 \%-77 \%)$, rhinorrhoea (76\%), myalgias $(40 \%-60 \%)$, headache $(22 \%-57 \%)$ and gastrointestinal symptoms, such as nausea, vomiting or diar- 
rhoea $(22 \%-39 \%)(7,8,20,32,37,39)$. Diarrhoea as a presenting symptom seems to occur more frequently in children than in adults (39), with up to $80 \%$ of children below the age of 14 years affected (7). Although gastrointestinal symptoms are unusual as signs of influenza, the frequency in the current pandemic is similar to previously described triplereassortant swine influenza virus cases (40). The median time from onset of symptoms to hospital admission was between 3 and 6 days, and 1 day from hospitalisation to ICU admission (7, 8, 37, 39).

\section{Hospitalisation}

From countries in the southern hemisphere, $23.6 \%-30.6 \%$ of reported cases required hospital admission, of which $11.7 \%-18.5 \%$ were admitted to the ICU $(3.6 \%-4.4 \%$ of the total number of cases) $(35,36)$. In contrast, northern hemisphere countries reported between $9 \%$ and $20.3 \%$ of confirmed pandemic $\mathrm{H} 1 \mathrm{~N} 1 / 09$ cases to require hospitalisation, of which $19 \%-36 \%(2 \%-3.9 \%$ of the total number of cases) required ICU admission (32, 37, 39).

Children accounted for $32 \%-45 \%$ of all admissions (36, 39 ), and had a marginally shorter median duration of admission compared to adults ( 3 vs. 4 days) (36). Hospitalisation rates were slightly higher in females than in males (24.3/ 100,00 and 20.9/100,000, respectively) (35), which resulted in $52 \%-67 \%$ of all admitted patients being female $(32,38)$ potentially explained by the higher risk conferred by pregnancy.

Hypertension (17\%), diabetes mellitus (17\%) and exacerbation of asthma or chronic obstructive pulmonary disease $(11 \%-14 \%)$ were commonly identified as concomitant presenting conditions $(7,37,38)$. In a USA cohort, $40 \%$ of hospitalised patients had radiologic findings consistent with pneumonia, $66 \%$ of which had an underlying medical condition. Patients with pneumonia on chest radiograph were more likely to have received antibiotics (97\%) than antivirals (73\%). Less than $2 \%$ of hospitalised patients had positive blood cultures, mainly due to Escherichia coli, Streptococcus pneumoniae and group A streptococcus (39).

\section{Intensive care}

As stated previously, between $11.7 \%$ and $36 \%$ of hospital admissions required intensive care, and the majority required ventilatory assistance (36). Respiratory support in the form of invasive ventilation was required by $56 \%-81 \%$ of patients for a median of 8 days $(7,36-39)$. Twelve percent of ICU admissions required extra-corporeal membrane oxygenation (36). Between $50 \%$ and $59 \%$ of patients required vasopressive support during hospitalisation, and 28\%-69\% received corticosteroids $(7,8,37)$. In some cohorts, all patients requiring mechanical ventilation had chest radiograph findings consistent with a diagnosis of acute respiratory distress syndrome (7). Seventy-one percent of critically ill patients had bilateral infiltrates on chest radiograph (37), likely due to primary viral pneumonia/pneumonitis. This is the most common clinical finding in severe cases and is present in up to
$49 \%$ of cases $(9,38)$. In Australia and New Zealand, the number of ICU admissions due to influenza A infection was 15 times higher than that for viral pneumonitis in recent years (38).

Secondary bacterial pneumonia was found in $20.3 \%-$ $32.1 \%$ of critically ill patients, the majority being caused by Staphylococcus aureus (up to 44\%, including methicillinresistant strains) or Streptococcus pneumoniae and Group A Streptococcus (12\%) (8, 9, 32, 37, 38). A clinical diagnosis of sepsis was established in $31 \%$ of critically ill patients, while $95 \%$ of ICU patients received antibiotics (39).

\section{Impact on health services}

Despite the high ICU admission rates, ICU occupancy in New Zealand peaked at about $25 \%$ of national capacity (35), and $30 \%$ of New South Wales' ICU capacity was occupied by confirmed or suspected pandemic H1N1/09 (36). The greatest impact on ICU resources was estimated to occur 4-6 weeks following the first confirmed winter ICU admission, although the increased workload lasted several weeks (38). In Australia, emergency department presentations and ambulance calls were increased by $6.3 \%$ and $13 \%$, respectively, over the corresponding period for 2008 (36).

\section{Groups at increased risk}

According to the WHO, risk factors for severe illness due to pandemic H1N1/09 seem to be similar to those for seasonal influenza (Table 1), with particular emphasis on the following categories: children under the age of 2 years, pregnant women (especially in the 3rd trimester) and patients with chronic lung conditions, such as asthma and chronic obstructive pulmonary disease (9).

At the time of presentation, comorbidities were present in $67 \%-100 \%$ of hospitalised patients, with a median of two comorbidities in critically ill patients $(8,37-39)$. Underlying conditions were identified in up to $60 \%$ of children, which is higher than the reported rates for seasonal influenza $(37 \%-43 \%)(39,42,43)$. Data on the frequency of comorbid conditions in non-hospitalised patients are not available. Preexisting conditions considered as comorbidities were not

Table 1 Groups at increased risk of severe disease from pandemic H1N1/09 virus infection (41).

1. Infants and young children, in particular $<2$ years

2. Pregnant women

3. Persons of any age with chronic pulmonary disease (e.g., asthma, COPD)

3. Persons of any age with chronic cardiac disease (e.g., congestive cardiac failure)

4. Persons with metabolic disorders (e.g., diabetes)

5. Persons with chronic renal or hepatic disease, certain neurological conditions, haemoglobinopathies, or primary or secondary immunosuppressive conditions

6. Children receiving chronic aspirin therapy

7. Persons $>65$ years 
always defined, but investigators frequently used those included in clinical outcome scores, such as Acute Physiology and Chronic Health Evaluation II (APACHE II), Pediatric Risk of Mortality (PRISM) or Sequential Organ Failure Assessment (SOFA).

The most common comorbidities reported were chronic lung disease (up to $41 \%$, including asthma at $27 \%-29 \%$ ), obesity (22\%-36.2\%), hypertension (24\%-26\%) (34, $37-39$ ) and human immunodeficiency virus (HIV) infection (53\%) in some populations (34). Active tuberculosis was identified in $9.7 \%$ of fatal South African cases (34).

\section{Pregnancy}

During previous pandemics and seasonal influenza epidemics, pregnant women were at higher risk for influenza-related morbidity and mortality compared with non-pregnant women, with a $27 \%$ mortality rate in the 1918-1919 influenza pandemic. Pregnancy also accounted for $50 \%$ of influenzarelated deaths amongst women of reproductive age in the 1957-1958 pandemic (44). Influenza infection also accounted for $\sim 50 \%$ of all morbidity suffered by females in the third trimester of pregnancy over a 20 -year period in the USA (45). Up to $33 \%$ of pregnant patients present with comorbid conditions, most often asthma (21\%), although some studies have reported all pregnant patients to have been previously healthy $(39,46,47)$.

The estimated risk of admission for pandemic H1N1/09 virus infection early in the pandemic was four times greater for pregnant females than for the general population (46). Pregnant patients accounted for $7 \%$ of hospital admissions and $7.7 \%-9.1 \%$ of ICU admissions, compared to the estimated pregnancy rate of $1 \%$ in the general population for some of the reporting countries $(32,37-39)$. Hospital admission rates may be inflated where clinicians have a lower threshold for admitting pregnant women (46). During previous seasonal influenza epidemics, females in the third trimester of pregnancy were three to four times more likely to require hospital admission for an acute cardiopulmonary condition compared with postpartum women (44). During the current pandemic, pregnant patients were more likely to present in the second $(17 \%-56 \%)$ or third trimester $(26 \%-67 \%)$ than in the first trimester $(9 \%-11 \%)(39,46)$.

The increased morbidity and mortality in pregnant patients may result from an altered immunological state, mechanical differences, such as decreased lung capacity, or physiologic changes, such as increased oxygen requirements (44). Pregnant patients may be exposed to the pandemic virus more frequently by living with young children, but it is unlikely that this accounts for all observed cases (47).

\section{Comorbidities}

Obesity has previously been associated with an increase in morbidity in critically ill patients, with a significantly higher rate of respiratory failure when compared to patients who were not overweight, but not consistently with mortality (48). Patients requiring admission during the current pandemic fre- quently had a higher body mass index (BMI) than the general population, and the mean BMI in one study was calculated at 34.6 (37). For hospitalised patients, between $29 \%$ and $36 \%$ were obese $(\mathrm{BMI}>30)$ and $14 \%-26 \%$ were severely obese (BMI $>40)$, which is higher than the estimated general prevalence from corresponding countries (8, 37-39). There was, however, no significant difference in BMI demonstrated between ICU non-survivors and survivors (37). Studies reported up to $90 \%$ of obese patients and $67 \%$ of obese children presented with underlying medical conditions (39), which may be confounding factors.

Little data have been published on the contribution of HIV towards pandemic H1N1/09-related morbidity or mortality. Fifty-three percent of confirmed fatalities in South Africa that were tested for HIV infection were found to be HIVpositive (34). It is expected that HIV infected patients may be at greater risk for rapidly progressing disease or secondary bacterial infections, and early empiric treatment with neuraminidase inhibitors are emphasised (49). Generally, guidelines for testing, treatment and chemoprophylaxis are similar to other groups that are at risk for complications of influenza infection. Patients with HIV are one of the target groups for the pandemic H1N1/09 vaccine and seasonal vaccine, and should receive the injectable inactivated form of either vaccine (49).

\section{Health care workers}

Health care workers appear to be at risk of contracting pandemic H1N1/09 infection if proper infection control measures are not adhered to. An early case report from Mexico reported that $12 \%$ of health care workers caring for influenza cases developed respiratory symptoms, although not all were confirmed to be pandemic H1N1/09 (7). After infection control measures were implemented, documented transmission decreased dramatically to between $0 \%$ and $0.6 \%$ $(7,8)$.

\section{Mortality}

\section{Mortality rate}

During the early stages of the pandemic, the overall mortality rate from pandemic $\mathrm{H} 1 \mathrm{~N} 1 / 09$ infection was $7 \%$ in hospitalised patients (39), $41 \%$ in critically ill patients and up to $58 \%$ in ventilated patients $(7,8)$. Mortality rates reported early in a pandemic may be higher due to delayed initiation of antiviral therapy or delayed admission to the hospital.

In more recent case series, ICU mortality ranged from $16.9 \%$ to $28 \%(38,39)$, the majority of fatalities occurring within the first 28 days of intensive care (37). Median duration of ICU stay was shorter for non-survivors at 7-10 days compared with survivors (12-13.5 days) $(8,36,37)$. Interestingly, a higher percentage of patients admitted to ICU for seasonal flu in New Zealand and Australia demised compared with those admitted for pandemic H1N1/09, although 
the pandemic strain had a higher absolute number of fatalities (38).

Early in the pandemic, the case fatality rate (CFR) was estimated at $0.33 \%$, higher than for seasonal influenza (20). A study from New Zealand reported an estimated CFR of $0.005 \%$ based on general practice surveillance of influenzalike illness and reported fatalities in hospitalised patients (35). This is markedly lower than the estimated CFR of $2.0 \%$ for the 1918 pandemic in New Zealand (35), although it may increase during a possible second wave of the pandemic.

\section{Age}

The median age of fatal cases in the southern hemisphere, ranging from 39 to 58 years $(33,36)$, was higher than that reported from the northern hemisphere (26 years) (39). The mortality in children appears to be less than in older patients, as $8 \%$ of children in a Canadian case series died compared to $17.3 \%$ of the entire study population (37). The mean age of survivors was 15-30 years compared with the mean age of non-survivors which ranged from 34 to 42 years $(34,37)$ which was significant in the South African cohort $(\mathrm{p}<0.01)$ (34).

\section{Risk factors}

In Australia and New Zealand, three factors were independently associated with in-hospital death: invasive ventilation, any co-existing medical condition as defined by the APACHE II-scoring system, and older age at an odds ratio of 1.02 per year of age (38).

Between $68 \%$ and $88.5 \%$ of fatal cases had an underlying chronic medical condition $(36,39)$, with chronic lung disease $(33 \%)$, including asthma (17\%), and chronic cardiovascular disease $(23 \%)$ being the most common (36).

Fatalities during pregnancy followed a gestational distribution comparable to hospital admissions: $67 \%-85 \%$ of patients in the third trimester, $5 \%-17 \%$ in the second trimester and $10 \%$ in the puerperium $(34,46)$. In keeping with hospitalisation rates, pregnancy has been shown to confer a 7.7 times higher risk for death over that for the general population (47). In a South African study, $28.4 \%$ of all fatalities were pregnant, which accounted for $56 \%$ of all females of reproductive age (34). All recorded fatalities in pregnant females resulted from primary viral pneumonia with subsequent acute respiratory distress syndrome $(46,47)$. Of those who died in South Africa, $71.4 \%$ were HIV positive and $19 \%$ had concurrent active tuberculosis (34).

Patients who died were likely to have more severe illness and greater organ dysfunction at presentation, as assessed by the APACHE II or SOFA scores, compared with those who survived $(7,8,37)$. In some series, all patients who died had documented multiple organ failure with significantly higher rates of renal failure (83\% vs. $7 \%$ ) compared with survivors (7). Patients who died during hospitalisation were more likely to have refractory hypotension $(100 \%)$ than those who survived (14\%) (7).

\section{Laboratory diagnosis}

Various diagnostic methods are available to confirm influenza A virus infection in respiratory samples. Rapid antigen tests and direct immunofluorescence assays (DFA) do not distinguish between different influenza A virus subtypes, and therefore cannot confirm an isolate to be pandemic H1N1/ 09. Selected reverse-transcriptase polymerase chain reaction (RT-PCR) systems as well as viral culture together with complementary tests have the ability to subtype influenza A virus strains (50).

Rapid antigen tests generally have low sensitivity (11\%-69\%) for detecting pandemic H1N1/09 (50-54). The sensitivity of all rapid tests decreased markedly with decreasing virus titres in specimens (51). However, the reported specificity was consistently greater than $93 \%(50,52)$. DFA showed comparable specificity to rapid antigen tests $(99.6 \%$ vs. $99.5 \%$ ), but higher sensitivity (47.2\% vs. $21.2 \%$ ) (50). The positive predictive value of rapid antigen tests or DFA is generally high when specific influenza viruses are circulating in the community. Under these circumstances, a positive result indicates probable infection with the circulating strain, but a negative result does not rule out infection and requires further testing (55). Viral culture showed a sensitivity of $88.9 \%$ and a specificity of $100 \%$ in the diagnosis of pandemic H1N1/09 when compared with a combination of RT-PCR, DFA and rapid antigen tests (50).

RT-PCR is superior to other methods for the diagnosis of pandemic H1N1/09 infection, with high sensitivity (97.8\%) and specificity (100\%) when compared to a combination of rapid antigen tests, DFA and viral culture (50). The ability to subtype influenza A strains rapidly can save time and resources, and may be especially important when pandemic H1N1/09 and seasonal influenza strains co-circulate, posing a risk of intra-human reassortment (56).

Laboratory confirmation of suspected cases of pandemic H1N1/09 virus infection is particularly important at the beginning of outbreaks. Once pandemic H1N1/09 is known to be the predominant circulating strain in a community, patients with typical influenza-like illness can be diagnosed on the basis of clinical presentation alone, which has a high predictive value (Figure 2). Laboratory testing should be reserved for high-risk patients, unusual cases or those with severe or progressive illness, especially where it may influence clinical management, but initiation of antiviral therapy should never be delayed (55). As seen during the Severe Acure Respiratory Syndrome (SARS) outbreak in 2003 (57), laboratory testing plays a modest role in patient management during outbreaks.

\section{Antiviral treatment}

Pandemic H1N1/09 virus is thus far susceptible to neuraminidase inhibitors. Treatment with oseltamivir or zanamivir is recommended for all patients with confirmed or suspected pandemic $\mathrm{H} 1 \mathrm{~N} 1 / 09$ requiring hospitalisation, or for those at 


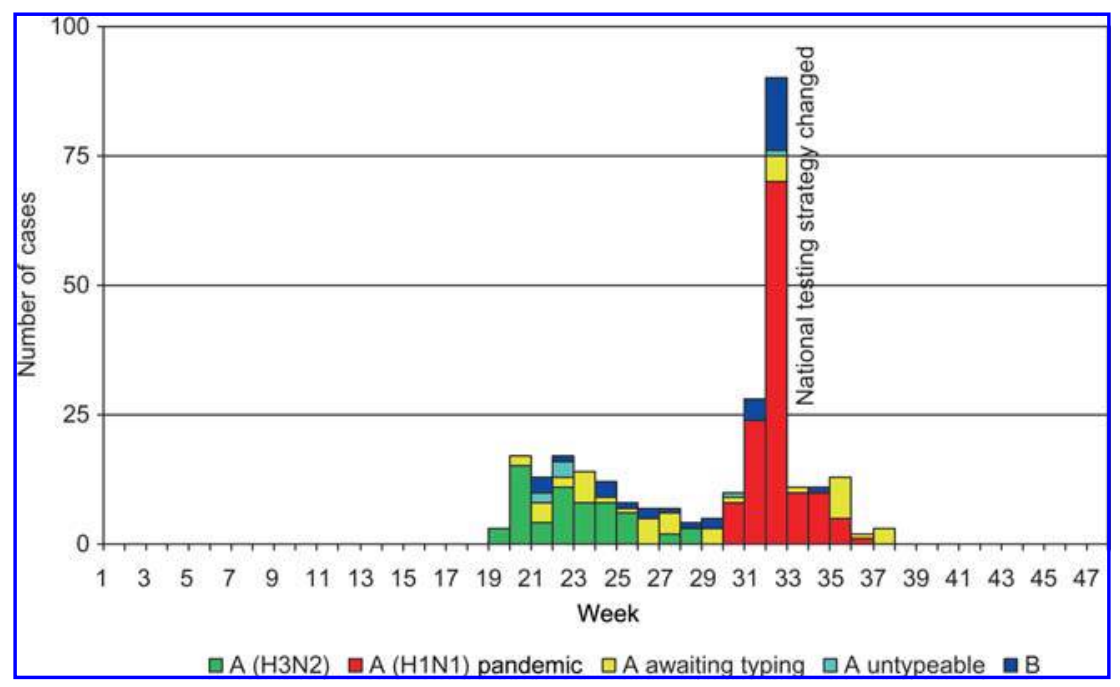

Figure 2 Influenza viruses isolated from samples sent by general practitioners participating in the influenza surveillance programme in the Western Cape Province, South Africa, from January until the end of October 2009.

During the first part of the southern hemisphere winter season, the majority of isolates were influenza A (H3N2), which was replaced by pandemic H1N1/09 virus as the dominant strain by week 31 . The national testing strategy was changed in week 32 , after the first 100 cases had been identified and sustained community transmission established.

higher risk of influenza related complications. The latter group includes children under the age of 2 years or adults older than 65 years, pregnant patients and those up to 2 weeks postpartum, people with chronic or immunosuppressive conditions and people younger than 19 years receiving long-term aspirin therapy (see also Table 1) (58). Antiviral chemoprophylaxis for seasonal influenza with oseltamivir or zanamivir has been shown to be effective among household contacts $(59,60)$, and is reserved for any of these high-risk groups or health care workers following contact with a confirmed or suspected case of pandemic H1N1/09, although early antiviral treatment is an emphasised alternative to prophylaxis (58). Oseltamivir may also be combined with amantadine or rimantadine if infection with either the seasonal or pandemic H1N1/09 strain is suspected (58). In severe cases, empiric antimicrobial therapy should be considered due to the high rate of secondary bacterial infection (9).

Neuraminidase inhibitors are effective when started within $48 \mathrm{~h}$ after onset of symptoms (58). In a multivariate analysis of hospitalised patients in the USA, receipt of antiviral drugs in the first 2 days of illness was the only variable significantly associated with a positive outcome (39). This is often difficult to achieve, as illustrated by a USA study which showed that therapy was initiated at a median of 3 days after onset of symptoms, with only $39 \%$ of hospitalised patients receiving antiviral therapy within $48 \mathrm{~h}$ (39). However, it has been suggested that oseltamivir therapy may still be beneficial in the treatment of seasonal influenza if initiated later (61), and may therefore be of benefit in pandemic H1N1/09 infection as well. During the current pandemic, patients who survived hospitalisation were 7.4 times (95\% CI, 1.8-31) more likely to have received neuraminidase inhibitors than those who died (8).

Oseltamivir, normally for use in persons 1 year and older, has been authorised by the USA Food and Drug Administration (FDA) for emergency use to treat pandemic H1N1/ 09 in infants below the age of 1 year, and for chemoprophylaxis in those older than 3 months (58). High dose oseltamivir (150 mg twice daily) and prolonged prophylaxis at normal treatment doses have been shown to be safe and well tolerated (62), and high dose treatment administered via nasogastric tube was shown to have a good pharmocokinetic profile in severe influenza A (H5N1) infection (63). No published data have shown high dose treatment to be more effective than treatment with normal doses (58). Oseltamivir is preferred over zanamivir for the treatment of pregnant women due to its systemic absorption $(62,64)$.

Treatment with intravenous peramivir, an unlicensed neuraminidase inhibitor, has been authorised by the FDA for emergency situations (58). Early treatment with a single dose of peramivir was found to be comparable to oral oseltamivir (65). Peramivir is indicated for use in adults and children that do not respond to oral or inhaled antiviral therapy, or when drug delivery by any other route than IV is not expected to be dependable (58). Emergency use approval has been granted for children of all ages, despite the fact that no paediatric patients received peramivir in clinical trials $(65,66)$.

\section{Antiviral drug resistance}

All pandemic H1N1/09 strains are intrinsically resistant to the M2 inhibitors amantadine and rimantadine due to the 
S31N mutation (serine to asparagine) in the M2 gene (32, 11). The earlier pandemic H1N1/09 isolates all tested sensitive to the neuraminidase inhibitors currently available (67). The first cases of oseltamivir-resistant pandemic H1N1/ 09 virus were identified in July 2009 (68). Oseltamivir resistance is conferred by the $\mathrm{H} 275 \mathrm{Y}$ mutation (histidine to tyrosine, $\mathrm{H} 274 \mathrm{Y}$ in $\mathrm{N} 2$ numbering) in the NA gene (69).

At the time of submission, less than 40 cases of oseltamivir resistant pandemic H1N1/09 strains have been reported. All isolates were shown to carry the $\mathrm{H} 275 \mathrm{Y}$ mutation, but remained sensitive to zanamivir. There is currently no evidence of sustained transmission or that these strains arose due to reassortment with other influenza viruses. Most of these strains were isolated from patients who either were on oseltamivir prophylaxis or received extended treatment courses of the drug, although three cases of oseltamivir resistance were reported in patients without a history of exposure (70).

Chemoprophylaxis associated resistance occurred predominantly as isolated events, and may be due to two possible mechanisms, First, viral mutation during low-level replication, possibly at subtherapeutic drug levels, or second, the resistant virus was acquired from an oseltamivir treated contact, although this has not been demonstrated (70).

Sixteen cases of treatment associated resistance have been reported to the WHO to date; seven of which occurred in immunosuppressed patients (70). Prolonged viral shedding increases the risk of developing drug resistance (70), and has been demonstrated in two immunocompromised individuals in the USA (71). Oseltamivir resistance should be considered in patients with protracted illness, particularly in the immunocompromised, and those who persistently test positive despite extended antiviral therapy. Patients who develop influenza-like illness despite receiving oseltamivir prophylaxis after exposure are also at a higher risk of developing resistance $(70)$.

\section{Vaccines}

There is no evidence that seasonal influenza vaccines, including adjuvant vaccines, produce significant levels of protection against the pandemic H1N1/09 strain $(19,29,31)$.

Inactivated- and live attenuated monovalent vaccines containing the A/California/7/2009 (H1N1) strain have been approved by the FDA, European Medicines Agency (EMEA) and other national and international regulatory bodies (72-74). Preliminary data indicate that the safety and immunogenicity profiles of the approved vaccines are similar to those of seasonal vaccines $(75,76)$.

The approved age groups for the vaccines differ by manufacturer and should be confirmed before administration of any vaccine. Generally, children under nine should receive two vaccine doses, $\sim 4$ weeks apart, as only $25 \%$ of children under the age of 36 months and $36 \%$ of those above 36 months showed a sufficient antibody response to a single dose of vaccine (72). As for seasonal influenza vaccine, pan- demic H1N1/09 vaccine is recommended for pregnant females at all stages of pregnancy (77). The vaccine should also reduce morbidity in the offspring, as seasonal influenza vaccine delivered to mothers has been shown to reduce influenza related morbidity by $63 \%$ in infants up to the age of 6 months (78).

\section{Outlook}

In contrast to many other zoonotic viruses that emerge unexpectedly (79), influenza pandemics can and must be expected. Despite years of pandemic preparation, most countries were poorly prepared for the current challenge. It is fortunately not a highly dangerous pathogen. However, even low mortality rates can result in large numbers of fatalities when few individuals have pre-existing immunity. This may also still change, as the new virus could adapt further to human hosts.

Antiviral treatment is widely available, although over use has occurred and some patients did not have access due to poor policies and implementation. Specific vaccines were developed rapidly, but immunisation programmes are challenged by major logistical and acceptance issues.

The future of pandemic H1N1/09 is uncertain. It may cause a second pandemic wave, as observed in previous pandemics, or it may replace or reassort with human seasonal A (H1N1) or A (H3N2) virus, or even with HPAI H5N1. It is, therefore, essential that comprehensive surveillance in human beings and animals is maintained to monitor the pandemic potential of influenza viruses.

\section{Highlights}

- Illness frequently occurs in previously healthy, young adults with a wide range of clinical presentations.

- The CFR of pandemic H1N1/09 is lower than that of previous influenza pandemics.

- Pregnant patients in the second or third trimesters, or early in the puerperium are at increased risk of developing severe disease.

- Primary viral pneumonia is the most common clinical finding in severe cases.

- Rapid antigen tests cannot confirm or exclude the diagnosis of pandemic H1N1/09 infection, and clinical diagnosis should be confirmed by RT-PCR in selected cases.

- The majority of circulating strains remain susceptible to the neuraminidase inhibitors.

- Live attenuated- and inactivated monovalent vaccines are available for indicated risk- and age groups.

\section{Acknowledgements}

South African National Institute of Communicable Diseases for subtyping of influenza A strains. 


\section{Conflict of interest}

Authors' conflict of interest disclosure: The authors stated that there are no conflicts of interest regarding the publication of this article.

Research funding: None declared.

Employment or leadership: None declared.

Honorarium: None declared.

\section{References}

1. World Health Organization. Influenza-like illness in the United States and Mexico, 24 April 2009. http://www.who.int/csr/don/ 2009_04_24/en/index.html.

2. World Health Organization. Transcript of statement by Margaret Chan, Director-General of the World Health Organization, 11 June 2009. http://www.who.int/mediacentre/influenzaAH1N1_ presstranscript_20090611.pdf.

3. World Health Organization. Transcript of Virtual Press Conference, 7 July 2009. http://www.who.int/mediacentre/Pandemic_ h1n1_presstranscript_2009_07_07.pdf.

4. World Health Organization. Pandemic influenza (H1N1) 2009 - Update 75. http://www.who.int/csr/don/2009_11_20a/en/ index.html.

5. Webby RJ, Webster RG. Emergence of influenza A viruses. Philos Trans R Soc Lond B Biol Sci 2001;356:1817-28.

6. World Health Organization. Cumulative Number of Confirmed Human Cases of Avian Influenza A/(H5N1) Reported to WHO. http://www.who.int/csr/disease/avian_influenza/country/cases_ table_2009_09_24/en/index.html.

7. Perez-Padilla R, de la Rosa-Zamboni D, Ponce de Leon S, Hernandez M, Quiñones-Falconi F, Bautista E, et al. INER Working Group on Influenza. Pneumonia and respiratory failure from swine-origin influenza A (H1N1) in Mexico. N Engl J Med 2009;361:680-9.

8. Domínguez-Cherit G, Lapinsky SE, Macias AE, Pinto R, Espinosa-Perez L, de la Torre A, et al. Critically ill patients with 2009 influenza A (H1N1) in Mexico. J Am Med Assoc 2009;302:1880-7.

9. World Health Organization. Clinical features of severe cases of pandemic influenza - pandemic (H1N1) 2009 briefing note 13. http://www.who.int/csr/disease/swineflu/notes/h1n1_clinical_ features_20091016/en/index.html.

10. Smith GJ, Vijaykrishna D, Bahl J, Lycett SJ, Worobey M, Pybus OG, et al. Origins and evolutionary genomics of the 2009 swine-origin H1N1 influenza A epidemic. Nature 2009; 459:1122-5.

11. Garten RJ, Davis CT, Russell CA, Shu B, Lindstrom S, Balish $\mathrm{A}$, et al. Antigenic and genetic characteristics of swine-origin 2009 A (H1N1) influenza viruses circulating in humans. Science 2009;325:197-201.

12. World Organization for Animal Health (OIE). Evolution of pandemic H1N1 2009 in animals. http://www.oie.int/eng/press/ en_091104.htm.

13. Neumann G, Noda T, Kawaoka Y. Emergence and pandemic potential of swine-origin H1N1 influenza virus. Nature 2009; 459:931-9.

14. Matrosovich M, Stech J, Klenk HD. Influenza receptors, polymerase and host range. Rev Sci Tech 2009;28:203-17.

15. Itoh Y, Shinya K, Kiso M, Watanabe T, Sakoda Y, Hatta M, et al. In vitro and in vivo characterization of new swine-origin H1N1 influenza viruses. Nature 2009;460:1021-5.
16. Maines TR, Jayaraman A, Belser JA, Wadford DA, Pappas C, Zeng $\mathrm{H}$, et al. Transmission and pathogenesis of swine-origin 2009 A (H1N1) influenza viruses in ferrets and mice. Science 2009;325:484-7.

17. Munster VJ, de Wit E, van den Brand JM, Herfst S, Schrauwen EJ, Bestebroer TM, et al. Pathogenesis and transmission of swine-origin 2009 A (H1N1) influenza virus in ferrets. Science 2009;325:481-3.

18. Han K, Zhu X, He F, Liu L, Zhang L, Ma H, et al. Lack of airborne transmission during outbreak of pandemic (H1N1) 2009 among tour group members, China, June 2009. Emerg Infect Dis 2009;15:1578-81.

19. Mauad T, Hajjar LA, Callegari GD, da Silva LF, Schout D, Galas FR, et al. Lung pathology in fatal novel human influenza A (H1N1) infection. Am J Respir Crit Care Med 2009. doi:10.1164/rccm.200909-1420OC.

20. Munayco CV, Gomez J, Laguna-Torres VA, Arrasco J, Kochel TJ, Fiestas V, et al. Epidemiological and transmissibility analysis of influenza A $(\mathrm{H} 1 \mathrm{~N} 1) \mathrm{v}$ in a southern hemisphere setting: Peru. Euro Surveill 2009;14. pii: 19299. http://www. eurosurveillance.org/ViewArticle.aspx?ArticleId = 19299.

21. Nishiura H, Wilson N, Baker MG. Estimating the reproduction number of the novel influenza A virus (H1N1) in a southern hemisphere setting: preliminary estimate in New Zealand. $\mathrm{N} \mathrm{Z}$ Med J 2009;122:73-7.

22. Nishiura H, Castillo-Chavez C, Safan M, Chowell G. Transmission potential of the new influenza A (H1N1) virus and its age-specificity in Japan. Euro Surveill 2009;14. pii: 19227. http://www.eurosurveillance.org/ViewArticle.aspx ?ArticleId = 19227.

23. Chowell G, Miller MA, Viboud C. Seasonal influenza in the United States, France, and Australia: transmission and prospects for control. Epidemiol Infect 2008;136:852-64.

24. Odaira F, Takahashi H, Toyokawa T, Tsuchihashi Y, Kodama T, Yahata Y, et al. Assessment of secondary attack rate and effectiveness of antiviral prophylaxis among household contacts in an influenza A (H1N1)v outbreak in Kobe, Japan, May-June 2009. Euro Surveill 2009;14. pii: 19320. http:// www.eurosurveillance.org/ViewArticle.aspx?ArticleId = 19320.

25. World Health Organization. New influenza A (H1N1) virus: global epidemiological situation, June 2009. Weekly Epidemiological Record 2009;84:249-57. http://www.who.int/wer/ 2009/wer8425/en/index.html.

26. Centers for Disease Control and Prevention. Introduction and transmission of 2009 pandemic influenza A (H1N1) virus Kenya, June-July 2009. MMWR 2009;58:1143-6. http:// www.cdc.gov/mmwr/preview/mmwrhtml/mm5841a1.htm.

27. Ferguson NM, Cummings DA, Cauchemez S, Fraser C, Riley S, Meeyai A, et al. Strategies for containing an emerging influenza pandemic in Southeast Asia. Nature 2005;437:209-14.

28. Ferguson NM, Cummings DA, Fraser C, Cajka JC, Cooley PC, Burke DS. Strategies for mitigating an influenza pandemic. Nature 2006;442:448-52.

29. Hancock K, Veguilla V, Lu X, Zhong W, Butler EN, Sun H, et al. Cross-reactive antibody responses to the 2009 pandemic H1N1 influenza virus. N Engl J Med 2009;361:1945-52.

30. Kelly H, Grant K. Interim analysis of pandemic influenza (H1N1) 2009 in Australia: surveillance trends, age of infection and effectiveness of seasonal vaccination. Euro Surveill 2009; 14. pii: 19288. http://www.eurosurveillance.org/ViewArticle. aspx?ArticleId $=19288$.

31. Centers for Disease Control and Prevention. Serum crossreactive antibody response to a novel influenza A (H1N1) 
virus after vaccination with seasonal influenza vaccine. MMWR 2009;58:521-4. http://www.cdc.gov/mmwr/preview/ mmwrhtml/mm5819a1.htm.

32. Novel Swine-Origin Influenza A (H1N1) Virus Investigation Team. Emergence of a novel swine-origin influenza A (H1N1) virus in humans. N Engl J Med 2009;360:2605-15.

33. Gomez J, Munayco C, Arrasco J, Suarez L, Laguna-Torres V, Aguilar P, et al. Pandemic influenza in a southern hemisphere setting: the experience in Peru from May to September, 2009. Euro Surveill 2009;14. pii: 19371. http://www.eurosurveillance. org/ViewArticle.aspx?ArticleId = 19371.

34. Archer B, Cohen C, Naidoo D, Thomas J, Makunga C, Blumberg $\mathrm{L}$, et al. Interim report on pandemic H1N1 influenza virus infections in South Africa, April to October 2009: epidemiology and factors associated with fatal cases. Euro Surveill 2009; 14. pii: 19369. http://www.eurosurveillance.org/ViewArticle. aspx?ArticleId $=19369$.

35. Baker MG, Wilson N, Huang QS, Paine S, Lopez L, Bandaranayake D, et al. Pandemic influenza A (H1N1)v in New Zealand: the experience from April to August 2009. Euro Surveill 2009;14. pii: 19319. http://www.eurosurveillance.org/ ViewArticle.aspx?ArticleId = 19319.

36. New South Wales public health network. Progression and impact of the first winter wave of the 2009 pandemic H1N1 influenza in New South Wales, Australia. Euro Surveill 2009; 14. pii: 19365. http://www.eurosurveillance.org/ViewArticle. aspx?ArticleId $=19365$.

37. Kumar A, Zarychanski R, Pinto R, Cook DJ, Marshall J, Lacroix J, et al. Canadian critical care trials group H1N1 collaborative. Critically ill patients with 2009 influenza A (H1N1) infection in Canada. J Am Med Assoc 2009;302:1872-9.

38. ANZIC Influenza Investigators. Critical care services and 2009 H1N1 influenza in Australia and New Zealand. N Engl J Med 2009;361:1925-34.

39. Jain S, Kamimoto L, Bramley AM, Schmitz AM, Benoit SR, Louie J, et al. 2009 Pandemic influenza A (H1N1) virus hospitalizations investigation team. Hospitalized patients with 2009 H1N1 influenza in the United States, April-June 2009. N Engl J Med 2009;361:1935-44.

40. Shinde V, Bridges CB, Uyeki TM, Shu B, Balish A, Xu X, et al. Triple-reassortant swine influenza A (H1) in humans in the United States, 2005-2009. N Engl J Med 2009;360:2616-25.

41. World Health Organization. Clinical management of human infection with pandemic (H1N1) 2009: revised guidance. http:// www . who.int/csr/resources / publications/swineflu/clinical_ management_h1n1.pdf.

42. Keren R, Zaoutis TE, Bridges CB, Herrera G, Watson BM, Wheeler $\mathrm{AB}$, et al. Neurological and neuromuscular disease as a risk factor for respiratory failure in children hospitalized with influenza infection. J Am Med Assoc 2005;294:2188-94.

43. Ampofo K, Gesteland PH, Bender J, Mills M, Daly J, Samore $\mathrm{M}$, et al. Epidemiology, complications, and cost of hospitalization in children with laboratory-confirmed influenza infection. Pediatrics 2006;118:2409-17.

44. Jamieson DJ, Theiler RN, Rasmussen SA. Emerging infections and pregnancy. Emerg Infect Dis 2006;12:1638-43.

45. Neuzil KM, Reed GW, Mitchel EF, Simonsen L, Griffin MR. Impact of influenza on acute cardiopulmonary hospitalizations in pregnant women. Am J Epidemiol 1998;148:1094-102.

46. Jamieson DJ, Honein MA, Rasmussen SA, Williams JL, Swerdlow DL, Biggerstaff MS, et al. Novel Influenza A (H1N1) Pregnancy Working Group. H1N1 2009 influenza virus infection during pregnancy in the USA. Lancet 2009;374: 451-8.

47. Mangtani P, Mak TK, Pfeifer D. Pandemic H1N1 infection in pregnant women in the USA. Lancet 2009;374:429-30.

48. Sakr Y, Madl C, Filipescu D, Moreno R, Groeneveld J, Artigas $\mathrm{A}$, et al. Obesity is associated with increased morbidity but not mortality in critically ill patients. Intensive Care Med 2008; 34:1999-2009.

49. Centers for Disease Control and Prevention. Updated Interim Recommendations - HIV-Infected Adults and Adolescents. http://www.cdc.gov/h1n1flu/guidance_HIV.htm.

50. Ginocchio CC, Zhang F, Manji R, Arora S, Bornfreund M, Falk L, et al. Evaluation of multiple test methods for the detection of the novel 2009 influenza A (H1N1) during the New York city outbreak. J Clin Virol 2009;45:191-5.

51. Centers for Disease Control and Prevention. Evaluation of rapid influenza diagnostic tests for detection of novel influenza A (H1N1) virus - United States, 2009. MMWR 2009;58:826-9. http://www.cdc.gov/mmwr/preview/mmwrhtml/mm5830a2.htm.

52. Faix DJ, Sherman SS, Waterman SH. Rapid-test sensitivity for novel swine-origin influenza A (H1N1) virus in humans. $\underline{N}$ Engl J Med 2009;361:728-9.

53. Centers for Disease Control and Prevention. Performance of rapid influenza diagnostic tests during two school outbreaks of 2009 pandemic influenza A (H1N1) virus infection - connecticut, 2009. MMWR 2009;58:1029-32. http://www.cdc.gov/ mmwr/preview/mmwrhtml/mm5837a1.htm.

54. Drexler JF, Helmer A, Kirberg H, Reber U, Panning M, Müller M, et al. Poor clinical sensitivity of rapid antigen test for influenza A pandemic (H1N1) 2009 virus. Emerg Infect Dis 2009;15:1662-4.

55. Centers for Disease Control and Prevention. Interim Recommendations for Clinical Use of Influenza Diagnostic Tests During the 2009-10 Influenza Season. http://www.cdc.gov/ h1n1flu/guidance/diagnostic_tests.htm.

56. Panning M, Eickmann M, Landt O, Monazahian M, Olschläger S, Baumgarte S, et al. Detection of influenza A (H1N1)v virus by real-time RT-PCR. Euro Surveill 2009;14. pii: 19329. http://www.eurosurveillance.org/ViewArticle.aspx ?ArticleId = 19329.

57. Berger A, Drosten Ch, Doerr HW, Stürmer M, Preiser W. Severe acute respiratory syndrome (SARS) - paradigm of an emerging viral infection. J Clin Virol 2004;29:13-22.

58. Centers for Disease Control and Prevention. Updated Interim Recommendations for the Use of Antiviral Medications in the Treatment and Prevention of Influenza for the 2009-2010 Season. http://www.cdc.gov/H1N1flu/recommendations.htm.

59. Monto AS, Pichichero ME, Blanckenberg SJ, Ruuskanen O, Cooper C, Fleming DM, et al. Zanamivir prophylaxis: an effective strategy for the prevention of influenza types A and B within households. J Infect Dis 2002;186:1582-8.

60. Hayden FG, Belshe R, Villanueva C, Lanno R, Hughes C, Small I, et al. Management of influenza in households: a prospective, randomized comparison of oseltamivir treatment with or without postexposure prophylaxis. J Infect Dis 2004;189: 440-9.

61. McGeer A, Green KA, Plevneshi A, Shigayeva A, Siddiqi N, Raboud J, et al. Toronto invasive bacterial diseases network. Antiviral therapy and outcomes of influenza requiring hospitalization in Ontario, Canada. Clin Infect Dis 2007;45:1568-75.

62. Ward P, Small I, Smith J, Suter P, Dutkowski R. Oseltamivir (Tamiflu) and its potential for use in the event of an influenza pandemic. J Antimicrob Chemother 2005;55(Suppl 1):i5-i21. 
63. Taylor WR, Thinh BN, Anh GT, Horby P, Wertheim H, Lindegardh $\mathrm{N}$, et al. Oseltamivir is adequately absorbed following nasogastric administration to adult patients with severe H5N1 influenza. PLoS One 2008;3:e3410. doi:10.1371/journal.pone. 0003410 .

64. Centers for Disease Control and Prevention. Novel influenza A (H1N1) virus infections in three pregnant women - United States, April-May 2009. MMWR 2009;58:497-500. http:// www.cdc.gov/mmwr/preview/mmwrhtml/mm5818a3.htm.

65. Centers for Disease Control and Prevention. Emergency use authorization of peramivir IV - fact sheet for health care providers. http://www.cdc.gov/h1n1flu/eua/Final\%20HCP\%20Fact \%20sheet\%20Peramivir\%20IV_CDC.pdf.

66. Birnkrant D, Cox E. The emergency use authorization of peramivir for treatment of 2009 H1N1 influenza. N Engl J Med 2009. NEJM 10.1056/nejmp0910479.

67. Centers for Disease Control and Prevention. Update: drug susceptibility of swine-origin influenza A (H1N1) viruses, April 2009. MMWR 2009;58:433-5. http://www.cdc.gov/mmwr/ preview/mmwrhtml/mm5816a6.htm.

68. World Health Organization. Pandemic (H1N1) 2009 briefing note 1 - viruses resistant to oseltamivir (Tamiflu) identified. Weekly Epidemiological Record 2009;84:299-300. http:// www.who.int/wer/2009/wer8429/en/index.html.

69. Ives JA, Carr JA, Mendel DB, Tai CY, Lambkin R, Kelly L, et al. The H274Y mutation in the influenza A/H1N1 neuraminidase active site following oseltamivir phosphate treatment leave virus severely compromised both in vitro and in vivo. Antiviral Res 2002;55:307-17.

70. World Health Organization. Oseltamivir-resistant pandemic (H1N1) 2009 influenza virus, October 2009. Weekly Epidemiological Record 2009;84:453-8. http://www.who.int/wer/ 2009/wer8444/en/index.html.
71. Centers for Disease Control and Prevention. Oseltamivirresistant novel influenza A (H1N1) virus infection in two immunosuppressed patients - Seattle, Washington, 2009. MMWR 2009;58:893-6. http://www.cdc.gov/mmwr/preview/ mmwrhtml/mm5832a3.htm.

72. Centers for Disease Control and Prevention. Update on influenza A (H1N1) 2009 monovalent vaccines. MMWR 2009; 58:1100-1. http://www.cdc.gov/mmwr/preview/mmwrhtml/ mm5839a3.htm.

73. European Medicines Agency. Pandemic influenza (H1N1) website. http://www.emea.europa.eu/influenza/vaccines/home.htm.

74. Australian Government Therapeutic Goods Administration. TGA approves vaccine against pandemic (H1N1) 2009 influenza. http://www.tga.gov.au/alerts/medicines/h1n1vaccine.htm.

75. Greenberg ME, Lai MH, Hartel GF, Wichems CH, Gittleson C, Bennet J, et al. Response after one dose of a monovalent influenza A (H1N1) 2009 vaccine - preliminary report. $\underline{N}$ Engl J Med 2009;361. NEJM 10.1056/nejmoa0907413.

76. Talbot HK, Keitel W, Cate TR, Treanor J, Campbell J, Brady $\mathrm{RC}$, et al. Immunogenicity, safety and consistency of new trivalent inactivated influenza vaccine. Vaccine 2008;26:405761.

77. Centers for Disease Control. Use of influenza A (H1N1) 2009 monovalent vaccine - recommendations of the Advisory Committee on Immunization Practices (ACIP), 2009. MMWR 2009; 58(Early Release):1-8. http://www.cdc.gov/mmwr/preview/ mmwrhtml/rr58e0821a1.htm.

78. Zaman K, Roy E, Arifeen SE, Rahman M, Raqib R, Wilson E, et al. Effectiveness of maternal influenza immunization in mothers and infants. N Engl J Med 2008;359:1555-64.

79. Ludwig B, Kraus FB, Allwinn R, Doerr HW, Preiser W. Viral zoonoses - a threat under control? Intervirology 2003;46:71-8. 